\title{
Orbital Evolution of Algol Binaries with a Circumbinary Disk
}

\author{
Wen-Cong Chen, Xiang-Dong Li \\ Department of Astronomy, Nanjing University, Nanjing 210093, China \\ and \\ Sheng-Bang Qian \\ National Astronomical Observatories/Yunnan Astronomical Observatory, Chinese Academy of Sciences, \\ P.O. Box 110, Kunming 650011, China
}

\begin{abstract}
It is generally thought that conservative mass transfer in Algol binaries causes their orbits to be wider, in which the less massive star overflows its Roche-lobe. The observed decrease in the orbital periods of some Algol binaries suggests orbital angular momentum loss during the binary evolution, and the magnetic braking mechanism is often invoked to explain the observed orbital shrinkage. Here we suggest an alternative explanation, assuming that a small fraction of the transferred mass forms a circumbinary disk, which extracts orbital angular momentum from the binary through tidal torques. We also perform numerical calculations of the evolution of Algol binaries with typical initial masses and orbital periods. The results indicate that, for reasonable input parameters, the circumbinary disk can significantly influence the orbital evolution, and cause the orbit to shrink on a sufficiently long timescale. Rapid mass transfer in Algol binaries with low mass ratios can also be accounted for in this scenario.
\end{abstract}

Subject headings: stars: general — binaries: close — stars: mass loss — stars: evolution — circumstellar matter

\section{Introduction}

An Algol binary is a semidetached binary system consisting of (1) an early type, main sequence primary component which does not fill its Roche lobe, and (2) a lobe-filling, less massive star that is substantially above the main sequence. The less massive star is cooler, fainter and larger (Giuricin \& Mardirossian 1981; Peters 2001). It is believed that star (2) is initially more massive, and evolves first to overflow its Roche lobe to transfer mass to star (1). After the rapid mass exchange, the lobefilling star (2) becomes less massive in the Algol binary stage.

The evolution of Algol binaries has been investigated extensively (e.g., Plavec 1968; Paczynski 1971; Refsdal \& Weigert 1969). Although conservative mass transfer seems to roughly reproduce the observed characteristics of a considerable frac- tion of Algol binaries (e.g. Nelson \& Eggleton 2001; De Loore \& van Rensbergen 2004), nonconservative evolution with mass and orbital angular momentum loss taken into account is required for better comparison between theories and observations (Thomas 1977; Refsdal et al. 1974; Sarna 1993). With regard to the orbital evolution, conservative mass transfer in Algol binaries leads to increase of the orbital periods because mass is transferred from the less massive star to the more massive component (Huang 1963). However, there is strong evidence showing that the orbital periods of some Algol binaries are actually decreasing (Qian 2000a,b, 2001a,b,c, 2002; Lloyd \& Guilbault 2002). Although it is uncertain whether the measured period changes are longterm (secular) changes or transient fluctuations, they clearly demonstrate that these systems must undergo mass and angular momentum loss during 
the evolution. Moreover, Qian (2002) find that the decreasing rates $\dot{P}_{\text {orb }}$ scale with the orbital periods $P_{\text {orb }}$ roughly as $-\dot{P}_{\text {orb }} \propto P_{\text {orb }}$.

This orbital decay may be explained by angularmomentum loss via magnetic stellar winds (Verbunt \& Zwaan 1981). Sarna, Muslimov, \& Yerli (1997) and Sarna, Yerli, \& Muslimov (1998) proposed that dynamo action can occur in masslosing stars in Algol-type binaries, and produce large-scale magnetic fields, which lead to magnetic braking of the stars. However, there could exist some potential difficulties for the magnetic braking mechanism (see below). Here we suggest an alternative interpretation assuming that a small fraction of the transferred mass would form a circumbinary $(\mathrm{CB})$ disk surrounding the binary system (see also van den Heuvel 1994). Previous works (Taam \& Spruit 2001; Chen et al. 2006) have already indicated that the $\mathrm{CB}$ disk can efficiently remove the orbital angular momentum and accelerate the binary evolution in accreting white dwarf or neutron star binaries. We describe the input physics that is necessary for the evolution model in section 2. Numerically calculated results for the evolutionary sequences of Algol binaries in two cases are presented in section 3. We make brief discussion and conclude in section 4 .

\section{Input Physics}

We consider an Algol binary consisting of a lobe-filling, less massive star (of mass $M_{2}$ ), and an early type, more massive companion (of mass $M_{1}$ ). The total orbital angular momentum of the system in a circular orbit is

$$
J=a^{2} \mu \frac{2 \pi}{P_{\text {orb }}},
$$

where $a=\left(G M_{\mathrm{T}} P_{\text {orb }}^{2} / 4 \pi^{2}\right)^{1 / 3}$ is the binary separation and $\mu=M_{1} M_{2} / M_{T}$ is the reduced mass of the binary system, $G$, and $M_{\mathrm{T}}$ are the gravitational constant and the total mass of system, respectively. We neglect the spin angular momentum of the components because it is smaller compared with the total orbital angular momentum of the system. Similar to Rappaport et al. (1983), in the calculations we consider two types of mechanisms for orbital angular momentum loss from the binary system, which are described as follows.

\subsection{Magnetic Braking}

Verbunt \& Zwaan (1981) point out that single low-mass main-sequence stars will undergo braking by the coupling between the magnetic field and the stellar winds. The magnetic braking mechanism assumes that the ionized particles stream out along the magnetic field lines which come off the convection envelope of the stars. The loss of the specific angular momentum in the stellar winds is very large because the outflowing material is tied in the magnetic field lines to corotate with the stars out to a long distance ( $\gtrsim 5-10$ stellar radii) (Schatzman 1962). In an Algol binary, the angular momentum loss of the evolved star by magnetic braking cause it to spin down. However, the tidal forces of the system will continuously act to spin the evolved star up back into corotation with the orbital rotation. The spin up takes place with the expense of the orbital angular momentum. Hence magnetic braking indirectly carries away the orbital angular momentum of the binary system.

The mass loss due to the magnetic winds during the mass transfer is generally very small, so we can assume that the total mass is conserved, and the orbital evolution is connected to the angular momentum loss rate and the mass transfer rate as follows

$$
\frac{\dot{P}_{\text {orb }}}{P_{\text {orb }}}=\frac{3 \dot{J}_{\mathrm{MB}}}{J}-\frac{3 \dot{M}_{2}}{M_{2}}(1-q),
$$

where $q=M_{2} / M_{1}$ is the mass ratio, and $\dot{J}_{\mathrm{MB}}$ is the angular momentum loss rate due to magnetic braking,

$$
\dot{J}_{\mathrm{MB}} \simeq-3.8 \times 10^{-30} M_{2} R_{\odot}^{4}\left(R_{2} / R_{\odot}\right)^{\gamma} \omega^{3} \mathrm{dyn} \mathrm{cm},
$$

where $\gamma$ is a dimensionless parameter in the range from 0 to 4 , and $\omega$ is the angular velocity of the star (Rappaport et al. 1983). Since $q<1$ and $\dot{M}_{2}<0$, the second term on the right hand side of Eq. (2) always causes the orbit to increase. Orbital shrinkage occurs only when the magnitude of the first term is larger than that of the second one, i.e.,

$$
\frac{\dot{P}_{\text {orb }}}{P_{\text {orb }}} \sim \frac{3 \dot{J}_{\mathrm{MB}}}{J} .
$$

One can show that with the magnetic braking law of Rappaport et al. (1983), the orbital decay rates with Eq. (4) are several orders lower than the observed ones. Moreover, from Eqs. (3) and (4) we 
can derive the following relation,

$$
-\dot{P}_{\text {orb }} \propto P_{\mathrm{orb}}^{(2 \gamma-7) / 3},
$$

where we have used the relation for the radius of the lobe-filling star $R_{2}=0.462[q /(1+q)]^{1 / 3} a$ with $0.1 \lesssim q \lesssim 0.8$ (Paczynski 1971). As we can see, only when $\gamma \gtrsim 4$ can we have a positive correlation between $-\dot{P}_{\text {orb }}$ and $P_{\text {orb }}$. More recent studies based on observations of rapidly rotating stars show that the above magnetic braking form may be inadequate (Queloz 1998; Sills et al. 2000; Andronov, Pinsonneault, \& Sills 2003). Sills et al. (2000) instead propose an empirical angular momentum loss prescription

$$
\begin{aligned}
\dot{J}_{\mathrm{MB}}=-K_{w} \omega^{3}\left(\frac{r_{2}}{m_{2}}\right)^{1 / 2}, & \omega \leq \omega_{\text {crit }} \\
\dot{J}_{\mathrm{MB}}=-K_{w} \omega \omega_{\text {crit }}^{2}\left(\frac{r_{2}}{m_{2}}\right)^{1 / 2}, & \omega>\omega_{\text {crit }}
\end{aligned}
$$

where $K=2.7 \times 10^{47} \mathrm{gcm}^{2}, \omega_{\text {crit }}$ is the critical angular velocity at which the angular momentum loss rate reaches a saturated state, $r_{2}$ and $m_{2}$ are $R_{2}$ and $M_{2}$ in solar units, respectively. Kim \& Demarque (1996) suggest that $\omega_{\text {crit }}$ is inversely proportional to the convective turbulent timescale in the star at 200 Myr age,

$$
\omega_{\text {crit }}=\omega_{\text {crit }, \odot} \frac{\tau_{\odot}}{\tau} .
$$

The magnitude of $\dot{J}_{\mathrm{MB}}$ under this magnetic braking law is at least one order of magnitude smaller than that in the Rappaport et al. (1983) model with $\gamma=4$ (Andronov et al. 2003), suggesting that more efficient angular momentum loss mechanism may be required to account for the orbit decay in Algol binaries.

\subsection{CB Disk}

Once Roche lobe overflow occurs in semidetached binaries, some fraction of the transferred material may leave the system in various ways. The mass outflow in cataclysmic variables (CVs) was noticed by Warner (1987) from the studies of UV and optical resonance lines.

Mass loss can also lead to orbital decrease in mass exchanging binaries. We first consider the case that a fraction $\delta$ of the transferred matter is lost from the binary system, carrying away the specific angular momentum of the secondary star (Rappaport et al. 1983). The change rate of orbital period can be written as

$$
\frac{\dot{P}}{P}=-3 \frac{\dot{M}_{2}}{M_{2}}\left[1-q(1-\delta)-\frac{\delta}{1+q}-\frac{1}{3} \frac{\delta q}{1+q}\right] .
$$

To account for the secular decreasing of orbital period, the mass loss fraction $\delta$ from the binary system should satisfy

$$
\delta>\frac{3\left(1-q^{2}\right)}{3-2 q-3 q^{2}} .
$$

For $q=0.5$, it requires $\delta>1.8$. Apparently this is impossible. Hence angular momentum loss via simple mass loss cannot interpret the orbital shrinking of some Algol binaries with low mass ratio.

In a theoretical view, Spruit \& Cao (1994) proposed that part of the outflow may be in the form of a slow wind near the orbital plane. It has been argued that during mass exchange the lost matter may form a disk structure surrounding the binary system rather than leave the binary system (van den Heuvel \& de Loore 1973; van den Heuvel 1994). More recently, Taam \& Spruit (2001) and Spruit \& Taam (2001) suggested that a Keplerian CB disk could be formed as a result of mass outflow in CVs. The CB disk can extract the orbital angular momentum from th binary orbit through tidal torques.

Since the mass transfer processes are similar in $\mathrm{CVs}$ as in Algol binaries, we assume that a constant fraction $\delta$ of the transferred mass feeds into the CB disk surrounding the Algol system, and $\dot{M}_{1}=-(1-\delta) \dot{M}_{2}$ (if the mass transfer rate is sub-Eddington). Same as in the standard thin accretion disks, the viscous torque in the CB disk is (Shakura \& Sunyaev 1973)

$$
T=-2 \pi r \nu \Sigma r \frac{d \Omega}{d r} r
$$

where $\nu$ is the viscosity, $r$ the radius, $\Sigma$ the surface density, and $\Omega=\left[G\left(M_{1}+M_{2}\right) / r^{3}\right]^{1 / 2}$ the local orbital angular velocity in the disk.

Assume that the gravitational interaction of the binary with the disk occurs locally at the inner edge $r_{\mathrm{i}}$ of the $\operatorname{disk}^{1}$, angular momentum from the

\footnotetext{
${ }^{1}$ In the following we use the subscript i to denote quantities evaluated at $r_{\mathrm{i}}$
} 
binary feeds into the disk at a rate proportional to the surface density $\Sigma_{\mathrm{i}}$. The viscous torque exerted by the CB disk on the binary can be shown to be (Taam \& Spruit 2001; Spruit \& Taam 2001)

$$
T_{\mathrm{i}} \equiv \dot{J}_{\mathrm{CB}}=\gamma\left(\frac{2 \pi a^{2}}{P_{\mathrm{orb}}}\right) \delta \dot{M}_{2}\left(\frac{t}{t_{\mathrm{vi}}}\right)^{p}
$$

where $\gamma^{2}=r_{\mathrm{i}} / a, t$ is the mass transfer time, and $p$ is a parameter determined by the viscosity. Suppose $\nu$ is the function of $r$ and $\Sigma$ with the following form (Spruit \& Taam 2001)

$$
\nu=\nu_{\mathrm{i}}\left(\frac{r}{r_{\mathrm{i}}}\right)^{n}\left(\frac{\Sigma}{\Sigma_{\mathrm{i}}}\right)^{m}
$$

then $p=(m+1) /[2(2 m+2-n)]$. In the following calculations we set $m=n=1$, so $p=1 / 3$.

By simple algebra, from the above equations we obtain the angular momentum loss rate via the CB disk

$$
\dot{J}_{\mathrm{CB}}=\delta\left(\frac{t}{t_{\mathrm{vi}}}\right)^{1 / 3} \dot{M}_{2} j_{\mathrm{CB}}
$$

where

$$
j_{\mathrm{CB}}=\gamma \frac{J}{\mu} .
$$

is the specific orbital angular momentum of the disk material at $r_{\mathrm{i}}$.

The viscous timescale $t_{\mathrm{vi}}$ at the inner edge of disk is

$$
t_{\mathrm{vi}}=\frac{4 r_{\mathrm{i}}^{2}}{3 \nu_{\mathrm{i}}}
$$

and $\nu_{\mathrm{i}}$ is given with the standard $\alpha$ prescription (Shakura \& Sunyaev 1973)

$$
\nu_{\mathrm{i}}=\alpha_{\mathrm{SS}} c_{\mathrm{s}} H_{\mathrm{i}},
$$

where $\alpha_{\mathrm{SS}}, c_{\mathrm{s}}, H_{\mathrm{i}}$ are the viscosity parameter (in the following calculations we set $\alpha_{\mathrm{SS}}=0.01$ ), the sound speed, and the scale height of disk at $r_{\mathrm{i}}$, respectively. Assuming that the disk is hydrostatically supported and geometrically thin with $H_{\mathrm{i}} / r_{\mathrm{i}} \sim 0.03$ (Belle et al. 2004), we have

$$
c_{\mathrm{s}} \simeq \Omega_{\mathrm{K}} H_{\mathrm{i}} .
$$

If we consider angular momentum loss only via the $\mathrm{CB}$ disk, the changing rate of the orbital period is then

$$
\frac{\dot{P}}{P}=-3 \frac{\dot{M}_{2}}{M_{2}}\left[1-\gamma \eta(1+q)-q(1-\delta)-\frac{1}{3} \frac{\delta q}{1+q}\right],
$$

where $\eta=\delta\left(t / t_{\mathrm{vi}}\right)^{1 / 3}$. Since $\dot{M}_{2}<0$, the orbital period will decrease when

$$
\eta>\frac{1-q(1-\delta)-(1 / 3) \delta q /(1+q)}{\gamma(1+q)} .
$$

For conservative evolution, $\delta=\eta=0$, Eq. (19) recovers to $q>1$ as expected.

In Fig. 1 we plot $\eta$ against $q$ in the solid curve when the orbital period is constant and $\delta \ll 1$. Here we take $r_{\mathrm{i}} / a=\gamma^{2}=1.7$ (Artymowicz \& Lubow 1994). Figure 1 shows that the CB disk presents a plausible mechanism removing the orbital angular momentum to explain the decrease of the orbital periods for reasonable values of $\eta$ (or $\delta$ ). Generally a larger $\eta$ is required for a smaller $q$. The dotted and dashed lines show two examples of Algol binary evolution, revealing how the orbital period evolution depends on the initial parameters. As seen from Eq. (14), the larger orbit, the larger angular momentum loss rate via the CB disk. This naturally results in a positive correlation between $\dot{P}_{\text {orb }}$ and $P_{\text {orb }}$.

\section{Numerical Results}

In this section we present the results of numerical calculations of the Algol binary evolutions in two typical cases. We have adopted an updated version of the evolution code developed by Eggleton $(1971,1972)$ (see also Han et al. 1994; Pols et al. 1995). We consider both the angular momentum loss mechanisms described in section 2, adopting the modified magnetic braking law (Eqs. [6][7]) for evolved stars with mass $\lesssim 1.5 M_{\odot}$. We take solar chemical compositions $(X=0.7, Y=0.28$, and $Z=0.02$ ) for both stars, and the ratio of the mixing length to the pressure scale height to be 2.0 .

(1) Initial masses $\left(M_{2}, M_{1}\right)=(3.0,2.0) M_{\odot}$ and orbital period $P_{\mathrm{orb}, \mathrm{i}} \simeq 1.4$ days

In a proto-Algol system with initial orbital period $P_{\text {orb,i }}<\sim 1-2$ days, the more massive star will fill its Roche lobe when it is still on the main sequence. This kind of Case A binary evolution has been investigated extensively (e.g., Eggleton 2000). Here we calculate the evolutionary sequences for a binary with initial masses $M_{2}=3 M_{\odot}, M_{1}=2 M_{\odot}$, and the initial orbital period $P_{\text {orb }, \mathrm{i}} \simeq 1.4$ days, assuming that a small fraction $\delta(=0.01,0.005)$ of the transferred mass 
forms a CB disk surrounding the system. Figure 2 shows the mass ratio, the orbital period and the mass transfer rate as a function of the time of mass transfer. It is clear that the mass transfer proceeds initially on short $(\lesssim 1 \mathrm{Myr})$, thermal-timescale, then on a much longer (hundreds of Myr) nucleartimescale when $q<1$, this is why we usually observe less massive donor stars in Algol binaries. The orbital period first decreases until $q \sim 0.6$. Its further evolution depends on the effect of orbital angular momentum loss by the $\mathrm{CB}$ disk. When $\delta=0.005$ the evolution is similar as the conservative evolution with continuously increasing orbital period. When $\delta=0.01$ the angular momentum loss is strong enough that at $q \sim 0.25, P_{\text {orb }}$ turns to decrease. The second orbital decay phase occupies most of the mass transfer lifetime. In both cases the mass transfer rates have a peak values $\sim 3 \times 10^{-6} \mathrm{M}_{\odot} \mathrm{yr}^{-1}$ at the beginning of Roche lobe overflow, then last a plateau phase $\sim 100-200$ Myr. It is also noted that, in both cases the mass transfer rates reach a peak when $t \sim 150-300 \mathrm{yr}$, indicating that the CB disk-induced angular momentum loss begins accelerating the mass transfer. (The evolution of $\eta$ was plotted against the mass ratio $q$ with the dotted curve in Fig. 1.)

(2) Initial masses $\left(M_{2}, M_{1}\right)=(3.0,2.0) M_{\odot}$ and orbital period $P_{\mathrm{orb}, \mathrm{i}} \simeq 3.4$ days

The calculated results for a relative wide system with $P_{\text {orb }, \mathrm{i}} \simeq 3.4$ days and $\delta=0.032,0.015$ are presented in Fig. 3. Compared with those in Fig. 2, the mass transfer rates are much higher, and the evolutionary timescale is smaller by a factor more than 100, suggesting that these systems are less likely to be observed. For both values of $\delta$, the orbital period shows a transition from decrease to increase when $q \sim 0.2-0.4$. The phases of orbit shrinkage last about $20 \%$ of the total mass transfer lifetime. When $\delta=0.015$, the mass transfer rate somewhat fluctuates during the late stages of the evolution though we have smoothed the mass transfer rate for clarity.

The calculated evolutionary tracks in the HR diagram are plotted in Fig. 4. The bold curves denote the phases in which the orbital period decreases. For comparison with observations, we also show the locations of four Algol systems which have decreasing orbital periods and well-measured parameters summarized in Table 1. As shown in the figure, the calculated results are roughly in

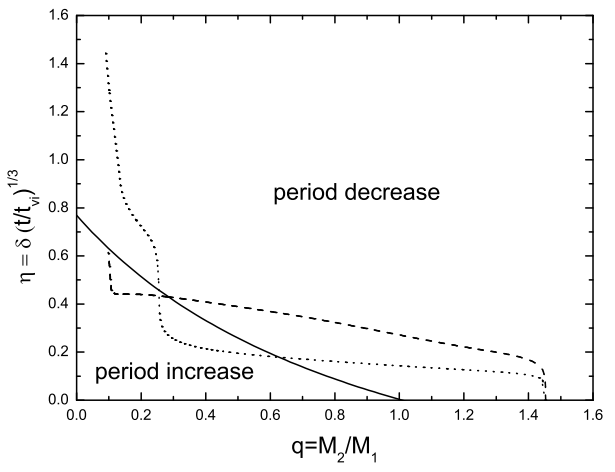

Fig. 1. - The figure of $\eta$ against the mass ratio $q$. The solid curve denote the division between period increase and decrease. The dashed and dotted curves correspond to the numerical results of Algol binary evolution with the initial orbital periods $P_{\text {orb }, \mathrm{i}}=3.4 \mathrm{~d}(\delta=0.032)$ and $1.4 \mathrm{~d}(\delta=0.01)$ and initial masses $\left(M_{2}, M_{1}\right)=(3.0,2.0) M_{\odot}$, respectively.
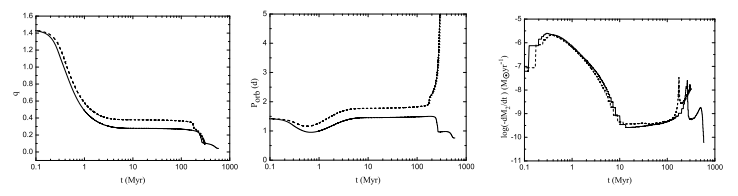

Fig. 2.- The evolution of the mass ratio $q$ (left), the orbital period $P_{\text {orb }}$ (center), and the mass transfer rate $\dot{M}$ (right) with the mass transfer time for a Algol binary with the initial orbital period $P_{\text {orb }, \mathrm{i}}=1.4 \mathrm{~d}$. The solid and dashed curves correspond to $\delta=0.01$ and 0.005 , respectively. The mass transfer rate have been slightly smoothed for clarity.
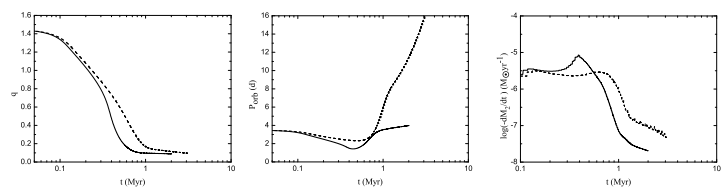

Fig. 3.- Same as Fig. 2, but for the initial orbital period $P_{\mathrm{orb}, \mathrm{i}}=3.4 \mathrm{~d}$. The solid and dashed curves correspond to $\delta=0.032$ and 0.015 , respectively. The mass transfer rate have been slightly smoothed for clarity. 
accordance with the observations, suggesting that they may represent the possible evolutionary sequences that lead to the formation of these systems. Figure 5 compares the calculated results of Algol binary evolution with the observational data in the $T_{\text {eff }}-P_{\text {orb }}$ plane. It seems that our model is also compatible with the observed spectral types of the four systems.

\section{Discussion and Conclusions}

It is an open question why some of the Algol systems show orbital shrinkage while others not. Although it is generally believed that there must be orbital angular momentum loss during the evolution of these binaries, the appropriate mechanism(s) for angular momentum loss has not been verified. Based on theoretical model of Spruit \& Taam (2001) and Taam \& Spruit (2001), we suggest that a CB disk may play an important role in determining the orbital change in Algol systems. Note that our CB disk hypothesis does not exclude the effect of (magnetized) mass loss. On the contrary, the formation of the $\mathrm{CB}$ disk may be closely related to the mass loss processes in the binary evolution. The existence of the CB disks may be revealed by their infrared radiation, as in GG Tau (Roddier et al. 1996). The Monte-Carlo simulation by Wood et al. (1999) has reproduced the morphology of the near IR radiation which is in accord with the observations by Roddier et al. (1996).

In this paper we have calculated the evolutionary sequence for Algol binaries with typical masses and orbital periods, taking into account both magnetic braking and the CB disk. The detailed calculations indicate that the orbital evolution of Algol binaries can be significantly affected by the CB disk, especially for Case A mass transfer. With adequate values of $\delta$, it is possible to account for the decrease of the orbital periods in some Algol binaries. In addition, the existence of $\mathrm{CB}$ disk can accelerate the evolution and enhance the mass transfer rates, as already noticed in the evolutionary study of CVs (Taam \& Spruit 2001). This suggests a plausible explanation for the rapid mass transfer observed in a few Algol binaries with very low mass ratios (Qian et al. 2002).

Finally, we note that the magnitude of the mass feeding parameter $\delta$ is highly uncertain, let

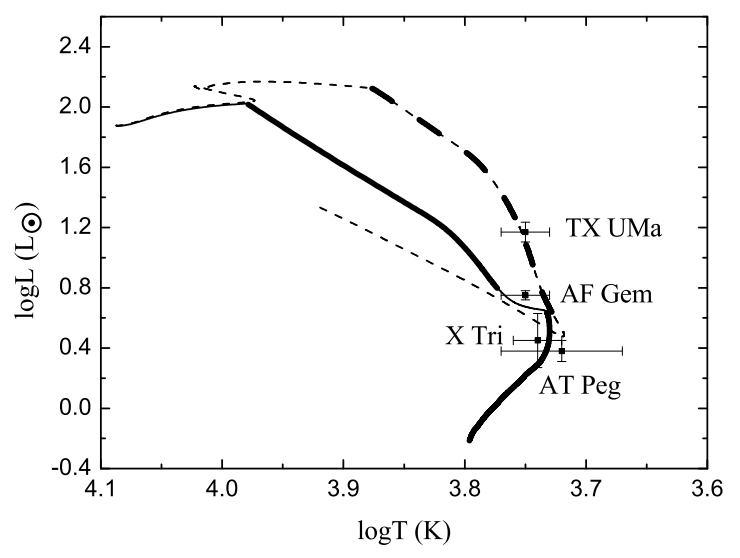

Fig. 4. - The calculated evolutionary tracks for Algol binaries in the HR diagram. The solid and dashed curves correspond to the initial orbital pe$\operatorname{riod} P_{\text {orb }, \mathrm{i}}=1.4 \mathrm{~d}(\delta=0.01)$ and $P_{\text {orb }, \mathrm{i}}=3.4 \mathrm{~d}$ $(\delta=0.032)$, respectively. The bold curves denote the phases in which the orbital period decreases. The filled rectangles represent the locations of the four Algol binaries listed in Table 1.

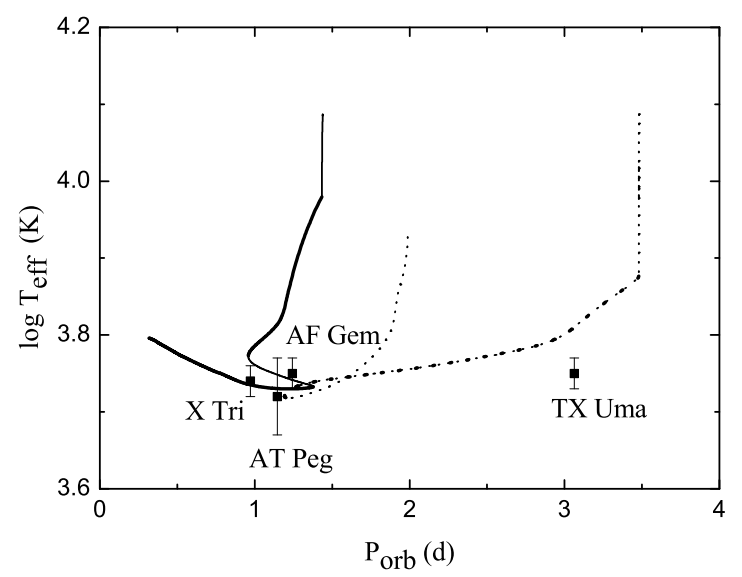

Fig. 5.- Evolutionary sequences in the donor star's effective temperature $T_{\text {eff }}$ versus orbital period $P_{\text {orb }}$ diagram. The solid and dotted curves correspond to the initial orbital period $P_{\text {orb }, \mathrm{i}}=1.4$ $\mathrm{d}(\delta=0.01)$ and $P_{\text {orb }, \mathrm{i}}=3.4 \mathrm{~d}(\delta=0.032)$, respectively. The bold curves denote the phases in which the orbital period decreases. The filled rectangles represent the locations of the four Algol binaries listed in Table 1. 
alone its variation with the mass transfer rates. These uncertainties make it difficult to present direct comparison between observations and theoretical predications for Algol binaries. However, our analysis provides a reasonable mechanism for angular momentum loss in semi-detached binaries including Algol binaries. More detailed multi-wavelength observations can provide stringent tests for the CB disk model, and the theories of the evolution of Algol binaries.

We thank the anonymous referee for his/her helpful comments improving this manuscript. This work was supported by Natural Science Foundation of China under grant number 10573010.

\section{REFERENCES}

Andronov, N., Pinsonneault, M., \& Sills, A. 2003, ApJ, 582, 358

Artymowicz, P., \& Lubow, S. H. 1994, ApJ, 421, 651

Belle, K. E., Sanghi, N., Howell, S. B., Holberg, J. B., \& Williams, P. T. 2004, AJ, 128, 448

Caughlan, G. R., \& Fowler, W. A. 1988, ADNAT, 40,284

Chen, W.-C, Li, X.-D., \& Wang, Z.-R. 2006, PASJ, 58, 153

De Loore, C. \& van Rensbergen,W. 2004, Ap\&SS, 296,353

Eggleton, P. P. 1971, MNRAS, 151, 351

Eggleton, P. P. 1972, MNRAS, 156, 361

Eggleton, P. P. 2000, New A, 44, 111

Giuricin, G., \& Mardirossian, F. 1981, ApJS, 46, 1

Han, Z., Podsiadlowski, P., \& Eggleton, P. P. 1994, MNRAS, 270, 121

Huang, S.-S. 1963, ApJ, 138, 471

Iglesias, C. A., Rogers, F. J., \& Wilson, B.G. 1992, ApJ, 397, 717

Itoh, N., Adachi, T., Nakagawa, M., Kohyama, Y., \& Munakata, H. 1989, ApJ, 339, 354
Kim, Y.-C., \& Demarque, P. 1996, ApJ, 457, 340

Li, X. -D. 2004, ApJ, 616, L119

Lloyd, G. \& Guilbault, P. 2002, The Observatory, 122,85

Maxted, P. F. L., Hill, G., \& Hilditch, R. W. 1994, A\&A, 285, 535

Maxted, P. F. L., \& Hilditch, R. W. 1995, A\&A, 301, 149

Maxted, P. F. L., Hill, G., \& Hilditch, R. W. 1995, A\&A, 301, 135

Mezzetti, M., Cester, B., Giuricin, G., \& Mardirossian, F. 1980, A\&AS, 39, 265

Nelson, C. A. \& Eggleton, P. P. 2001, ApJ, 552, 664

Paczyński, B. 1971, ARA\&A, 9, 183

Peters, G. 2001, Ap\&SS, 264, 79

Plavec, M. 1968, Adv. Astr. Ap., 6, 201

Pols, O., Tout, C. A., Eggleton, P. P., \& Han, Z. 1995, MNRAS, 274, 964

Qian, S.-B. 2000a, AJ, 119, 901

Qian, S.-B. 2000b, AJ, 119, 3064

Qian, S.-B. 2001a, AJ, 121, 1614

Qian, S.-B. 2001b, AJ, 122, 1561

Qian, S.-B. 2001c, AJ, 122, 2686

Qian, S.-B. 2002, PASP, 114, 650

Qian, S.-B. et al. 2002, A\&A, 396, 609

Queloz, D., Allain, S., Mermilliod, J. C., Bouvier, J., \& Mayor, M. 1998, A\&A, 335, 183

Rappaport, S., Verbunt, F., \& Joss, P. C. 1983, ApJ, 275, 713

Refsdal, S., Roth, M. L., \& Weigert, A., 1974, A\&A, 36, 113

Refsdal, S. \& Weigert, A., 1969, A\&A, 1, 16

Roddier, C., Roddier, F., Northcott, M. J., Graves, J. E., \& Jim, K. 1996, ApJ, 463, 326 
Sarna, M. 1993, MNRAS, 262, 534

Sarna, M., Muslimov, A., \& Yerli, S. K. 1997, MNRAS, 286, 209

Sarna, M., Yerli, S. K., \& Muslimov, A. 1998, MNRAS, 297, 760

Schatzman, E. 1962, AnAp, 25, 18

Shakura, N. I., \& Sunyaev, R. A. 1973, A\&A, 24, 337

Sills, A., Pinsonneault, M. H., \& Terndrup, D. M. 2000, ApJ, 534, 335

Spruit, H. C., \& Cao, X. 1994, A\&A, 287, 80

Spruit, H. C., \& Taam, R. E. 2001, ApJ, 548, 900

Taam, R. E., \& Spruit, H. C. 2001, ApJ, 561, 329

Thomas, M. C. 1977, ARA\&A, 15, 127

van den Heuvel, E.P.J., \& de Loore, C. 1973, A\&A, 25, 387

van den Heuvel, E.P.J. 1994 in Interacting Binaries (Saas-Fee 22), Shore, S. N., et al., eds., p263

Verbunt, F., \& Zwaan, C. 1981, A\&A, 100, L7

Warner, B. 1987, MNRAS, 227, 23

Wood, K., Crosas, M., \& Ghez, A. 1999, ApJ, 516, 335

This 2-column preprint was prepared with the AAS LATEX macros v5.2. 
TABLe 1

Observed Parameters of four Algol System

\begin{tabular}{ccccccccc}
\hline \hline Source & $P(\mathrm{~d})$ & $\dot{P}\left(10^{-6} \mathrm{~d} \mathrm{yr}^{-1}\right)$ & Spectrum & $M_{1}\left(M_{\odot}\right)$ & $M_{2}\left(M_{\odot}\right)$ & $\log L_{1}\left(L_{\odot}\right)$ & $\log L_{2}\left(L_{\odot}\right)$ & References \\
\hline X Tri & 0.9715397 & -0.142 & A2/G3 & $2.3 \pm 0.7$ & $1.2 \pm 0.3$ & $1.21 \pm 0.06$ & $0.45 \pm 0.18$ & 1 \\
AT Peg & 1.146082 & -0.638 & A4/G & $2.22 \pm 0.065$ & $1.05 \pm 0.025$ & $1.19 \pm 0.025$ & $0.38 \pm 0.07$ & 2 \\
AF Gem & 1.2435012 & -0.0992 & B9/G0 & $3.37 \pm 0.11$ & $1.155 \pm 0.038$ & $1.78 \pm 0.09$ & $0.75 \pm 0.03$ & 3 \\
TX UMa & 3.0632881 & -0.713 & B8/G0 & $4.76 \pm 0.16$ & $1.18 \pm 0.04$ & $2.30 \pm 0.04$ & $1.17 \pm 0.065$ & 4 \\
\hline
\end{tabular}

${ }^{a}$ References: 1 - Mezzetti et al. (1980), 2 - Maxted et al. (1994), 3 - Maxted \& Hilditch (1995), 4 - Maxted et al. (1995). 